\title{
A review of disaster and crisis
}

\begin{abstract}
A disaster and a crisis are two different, and related events. The two terms are sometimes used interchangeably. Man-made disaster that occurs at an industrial organization, may develop into an industrial crisis. Crisis can happen to any organization. It has been noted that there were no universally accepted definitions yet developed for disaster and crisis. There is also no universally available criteria, to define the disaster in terms of the consequences, such as the casualties and the cost of damage. This paper reviews the definitions, types, characteristics, criteria and models of disaster and crisis. Also the types of crisis were rearranged and the differences between the disasters and crises have been summarized.
\end{abstract}

Keyword: Disasters; Crisis; Model 